\title{
DÉTERMINATION DE LA ZONE OPTIMALE DE PRÉLÈVEMENT D'ÉCAILLES CHEZ LES SMOLTS DETRUITE DE MER (SALMO TRUTTA L.)
}

\author{
Dominique OMBREDANE (1), A. RICHARD (2)
}

(1) Laboratoire d'Écologie Hydrobiologique - INRA - 65, rue de St-Brieuc - 35042 RENNES Cedex

(2) Conseil Supérieur de la Pêche - DR2 - 84, rue de Rennes - 35110 CESSON-SEVIGNE

\section{RÉSUMÉ}

Les écailles de 34 juvéniles de Truite de mer (Salmo trutta L.) de $1^{\text {* }}$ et $2^{+}$ans ont été échantillonnées sur 12 parties du corps. Le taux de régénération en avant de la nageoire dorsale ou nettement au-dessus de la ligne latérale est plus faible ( 40 à $60 \%$ ) qu'ailleurs (65 à $75 \%$ ). De plus, les écailles y sont plus petites et plus arrondies.

La structure des écailles a été analysée sur 8 sites pour 14 individus. Au niveau de l'épaule, le nombre de circuli est significativement plus faible quelle que soit l'annee de croissance, et l'écart inter-circuli y est sensiblement plus petit qu'ailleurs. Les longueurs des poissons à 1 an, obtenues par rétrocalcul à partir des mensurations des écailles de l'épaule, avec le modèle isométrique de LEA, apparaissent sous-estimées.

Ces résultats suggèrent que la meilleure zone de prélèvement d'écailles pour les jeunes truites de mer se situe dans les 3 premiers rangs en-dessous ou au-dessus de la ligne latérale et de part et d'autre d'une droite joignant l'arrière de la nageoire dorsale et l'avant de la nageoire anale. Compte tenu d'un taux de régénération élevé, un prélèvement minimum de 10 à 15 écailles est recommandé.

\section{DETERMINATION OF OPTIMUM SCALES SAMPLING LOCATION FOR SEA TROUT SMOLTS (SALMO TRUTTA L.)}

\section{SUMMARY}

Scales of 34 sea trout (Salmo trutta L.) smolts, $1^{+}$and $2^{+}$, were sampled from 12 body areas. The proportion of regenerated scales removed before dorsal fin or far above the lateral line, is lower $(40$ to $60 \%$ ) than elsewhere (65 to $75 \%)$. From these areas, scales have smaller size and rounder form.

Scales structure is studied on 8 areas of 14 fishes. Scales located on shoulder have significant less circuli whatever the growth year and intercirculi space is lightly smaller. Back-calculated lengths at one year old from shoulder scales with LEA's model seem underestimated.

The results indicate that the better area of scales removing for young sea trout is located at rows 1-3 above and under lateral iine and on both sides of a line extending from the posterior edge of the dorsal fin to the anterior edge of the anal fin. As the regeneration rate is high, a minimum 10-15 scales sample is recommended.

\section{INTRODUCTION}

L'utilisation des écailles dans les études biologiques et écologiques des salmonidés présente un intérêt aujourd'hui largement reconnu. Aussi, les écailles sont utilisées depuis longtemps pour la détermination de l'âge et l'étude de la croissance tant pour la truite (Salmo trutta L.) que pour le Saumon atlantique (Salmo salar L.) (DAHL K., 1910, in BEALL et DAVAINE, 1988). Plus récemment, des analyses scalimétriques fines ont été mises en œuvre chez le Saumon atlantique (S. salar $L$.) dans le but d'appréhender plus précisément la croissance aux différents stades (LINDROTH, 1963; BAGLINIERE et MAISSE, 1985) ou encore de discriminer les stocks (REDDIN, 1981). II est alors rapidement apparu la nécessité de définir une zone standard de prélèvement des écailles (ANONYME, 1984; BAGLINIERE, 1985) compte tenu de la variabilité des caractéristiques scalaires sur le corps de cette espèce (ANON, 1982, in ANONYME, 1984 ; MARTYNOV, 1983). Des démarches similaires ont aussi été effectuées sur le genre Oncorhynchus (SCARNECCHIA, 1979; BILTON, 1984 ; etc.). 
Chez la truite commune (Salmo trutta L.), espèce étudiée depuis longtemps, les connaissances relatives à la forme migratrice anadrome se sont surtout développées dans les deux dernières décennies (DAVAINE et BEALL, 1982). Ainsi, en France, la truite de mer peut représenter une ressource intéressante, notamment dans les rivières du Nord-Ouest (RICHARD, 1986 ; FOURNEL et al, 1987). Sa gestion et son exploitation nécessitent alors un minimum de connaissances biologiques préalables.

Comme pour le Saumon, on assiste depuis peu chez la Truite de mer à une utilisation fine du matériel scalaire (comptage de circuli, interprétation des structures, rétromesures, etc.) pour:

- séparer les stocks sédentaires et migrateurs chez les adultes (BEALL, 1979; LE CREN, 1985; BEALL et DAVAINE, 1988).

- identifier les différents types de la forme migratrice d'un même bassin (BEALL, 1979; RICHARD, 1986).

- discriminer les stocks de Truite de mer adultes provenant de différents bassins (SYCH, 1983).

- déterminer l'âge, notamment l'âge d'eau douce (BEALL et DAVAINE, 1988).

- étudier la croissance (FAHY, 1983).

Or, pour cette espèce aucune zone standard n'a encore été définie. La nécessité de prélever les écailles en un site précis (quelle que soit l'espèce) afin d'éviter les erreurs tant dans la détermination de l'âge que dans des études de croissance a été mentionnée depuis très longtemps (HUNTSMAN, 1918 in HILE, 1970, JARVI et MENZIES, 1934; etc). II apparaît donc nécessaire de standardiser les prélèvements d'écailles également chez la truite, compte tenu du développement des analyses fines du matériel scalaire et pour permettre la confrontation des connaissances.

En outre, la description précise du site de prélèvement des écailles n'apparaît pas toujours dans les travaux portant sur l'espèce Salmo trutta. Les écailles sont généralement prélevées audessus de la ligne latérale, sur des sites localisés selon les cas entre l'avant de la nageoire dorsale et la nageoire adipeuse. L'épaule dans la partie antérieure du poisson a aussi été utilisée (KIPLING, 1962; EUZENAT et FOURNEL, comm. pers.). Certains auteurs prélèvent depuis peu sur la zone standard définie pour le Saumon (RICHARD, 1986) compte tenu de la similitude morphologique des deux espèces.

En vue de normaliser les prélèvements d'écailles pour l'espèce Salmo trutta, il est proposé d'effectuer une analyse de la variabilité des caractéristiques des écailles selon leur localisation sur le corps du poisson. Ce travail doit être réalisé tant chez les juvéniles, objet des résultats présentés ici, que chez les adultes (OMBREDANE et RICHARD, données non publiées). Pour étudier cette variabilité, sont retenus des critères de forme (LINDROTH, 1963; CARLANDER, 1985 ; BURDAK, 1986) de taille et de structure (SCARNECCHIA, 1979; MARTYNOV, 1983; BILTON, 1984). Le pourcentage d'écailles régénérées semble aussi important à étudier compte tenu du taux élevé de régénération (environ $60 \%$ ) observé chez des jeunes truites de mer (finnocks) (BAGLINIËRE et al, 1986). Ce critère a d'ailleurs été pris en compte pour le Saumon atlantique (ANONYME, 1984).

\section{MATÉRIEL ET MÉTHODES}

L'étude porte sur 34 juvéniles de Truite de mer, au stade smolt ou présmolt capturés par piègeage au printemps 1987, sur le Douet de la Taille, affluent de la Touques, rivière côtière de Basse Normandie. L'échantillon est composé de 13 individus âgés de $1^{+}$an et 21 individus de 2 ou $2^{+}$ans. Dans la mesure du possible, deux poissons ont été retenus par classe de $5 \mathrm{~mm}$ pour la gamme de taille $130-245 \mathrm{~mm}$.

\subsection{Les sites de prélèvement}

Le choix des sites de prélèvement d'écailles s'inspire en partie des études similaires faites chez le Saumon (SCARNECCHIA, 1979; MARTYNOV, 1983 ; BILTON, 1984 ; etc.) el des pratiques adoptées dans les différents travaux relatifs à la Truite. Au total 12 sites sont retenus (figure 1). Ils peuvent se regrouper en:

- 4 zones "verticales" de la partie antérieure vers la partie postérieure du poisson.

- Zone $A$ : centrée sur une ligne joignant l'insertion antérieure de la nageoire dorsale à celle de la nageoire pectorale (sites $A_{1}, A_{2}, A_{3}$ et E) (BILTON, 1984; PREVOST, 1987).

- Zone $B$ : centrée sur une ligne partant du milieu de la nageoire dorsale vers l'insertion antérieure de la nageoire ventrale (ALVORD, 1954 ; MARTYNOV, 1983 ; BILTON, 1984 ; PREVOST, 1987).

- Zone C : centrée sur une ligne joignant l'insertion postérieure de la nageoire dorsale à l'insertion antérieure de la nageoire anale (SYCH, 1967; MARTYNOV, 1983; BILTON, 1984; HESTHAGEN, $1985 ; \ldots$. . 

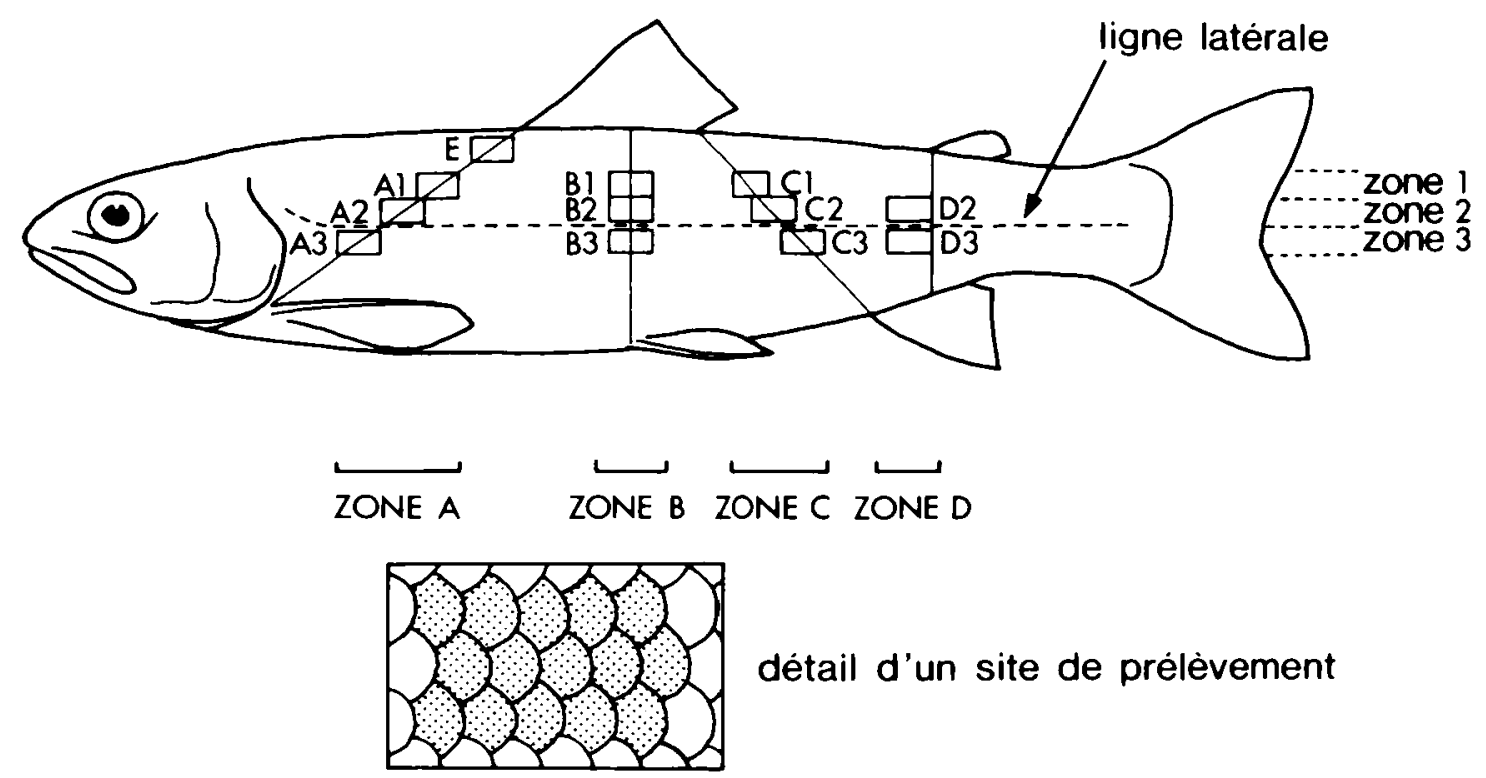

figure 1 : Localisation des sites de prélèvement d'écailles chez les smolts de truite de mer. Regroupement des sites en 3 zones horizontales (1, 2 et 3 ) et en 4 zones "verticales" (A, B, C, D). Détail d'un site de prélèvement.

figure 1 : Localization of the scales sampling areas on the smolt sea trouts. Areas are grouped in 3 horizontal zones $(1,2,3)$ and in 4 "vertical" zones (A, B, C, D). Detail of a sampling area.

- Zone D : en avant d'une droite partant de l'insertion antérieure de la nageoire adipeuse et perpendiculaire à la ligne latérale (BILTON, 1984 ; HESTHAGEN, 1985).

- 3 zones "horizontales" définies par rapport à la ligne latérale.

- Zone $1: 4^{\mathrm{e}}$ au $6^{\mathrm{e}}$ rang au-dessus de la ligne latérale.

- Zone 2 : $1^{\text {er }}$ au $3^{\text {e }}$ rang au-dessus de la ligne latérale.

- Zone 3 : $1^{\text {er }}$ au $3^{\text {e }}$ rang en-dessous de la ligne latérale.

Le site $E$, représentant l'épaule, est localisé dans la zone A entre le $13^{\mathrm{e}}$ et le $15^{\mathrm{e}} \mathrm{rang}$. Un tel site, dénommé aussi "épaule", a été analysé par PREVOST (1987) chez les adultes de Saumon atlantique.

\subsection{Choix des écailles et taux de régénération}

Pour chaque site, 15 écailles (figure 1) sont prélevées, sur le côté gauche du poisson, dans un rectangle contenant 5 écailles par rang (SCARNECCHIA, 1979) et 3 rangs (MARTYNOV, 1983). Dans les cas où 3 écailles exemptes de toute régénération ne sont pas disponibles, un prélèvement complémentaire est effectué sur le site homologue du côté droit du poisson. En effet, BILTON (1984) a montré que les écailles des deux côtés étaient équivalentes pour les analyses scalimétriques.

En se basant sur l'observation (figure 2) des 15 écailles ainsi prélevées sur le côtè gauche, on calcule pour chaque site:

- $t_{1}$ : pourcentage de bonnes écailles, ayant un nucléus petit et arrondi,

- t2 : pourcentage d'écailles peu régénérées, dont le nucléus est déformé, mais permettant une bonne détermination de l'âge,

- t3: pourcentage d'écailles largement régénérées, interdisant la détermination de l'âge.

\subsection{Caractéristiques des écailles}

Sur 3 bonnes écailles de chaque site, les mesures suivantes (figure 3 ) sont faites au grossissement $50 \times$ sur un projecteur de film (Projectina) muni d'un micromètre (500 gradations $=1 \mathrm{~cm}$ ) :

$L=$ le plus grand diamètre longitudinal (longueur)

$\mathrm{I}=$ le plus grand diamètre transversal (largeur) perpendiculaire à $L$

$\mathrm{R}=$ le plus grand rayon longitudinal (partie antérieure de l'écaille) passant par le nucléus

$r=$ le plus grand rayon transversal, perpendicalaire à $\mathbf{R}$ et passant par le nucléus. 


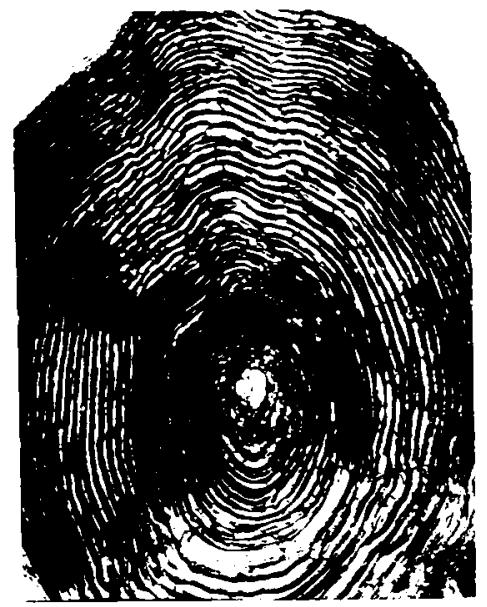

Ecat1le "bonne"

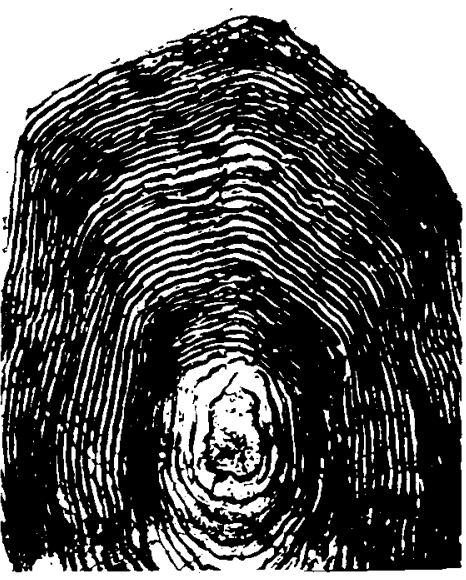

Ecaille peu régénérée

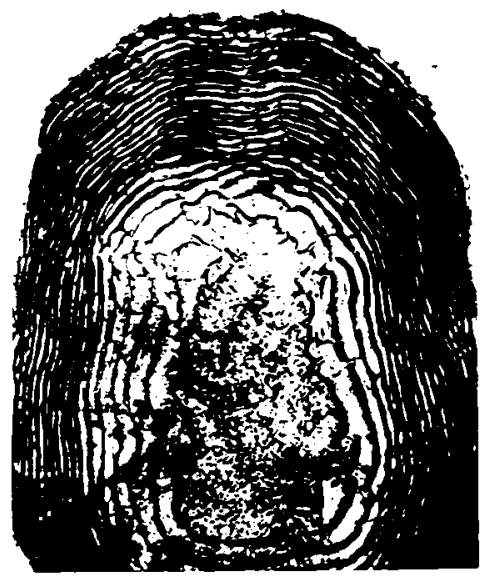

Ecallle régēnérée

figure 2 : Différents types d'écaille de smolts de truite de mer selon leur degré de régénération. Exemple d'un poisson âgé de $2^{+}$ans - longueur fourche $=221 \mathrm{~mm}$.

figure 2 : Various king of smolt sea trouts'scales with different degree of regeneration. Example of a 2 years old fish - fork length $=221 \mathrm{~mm}$.

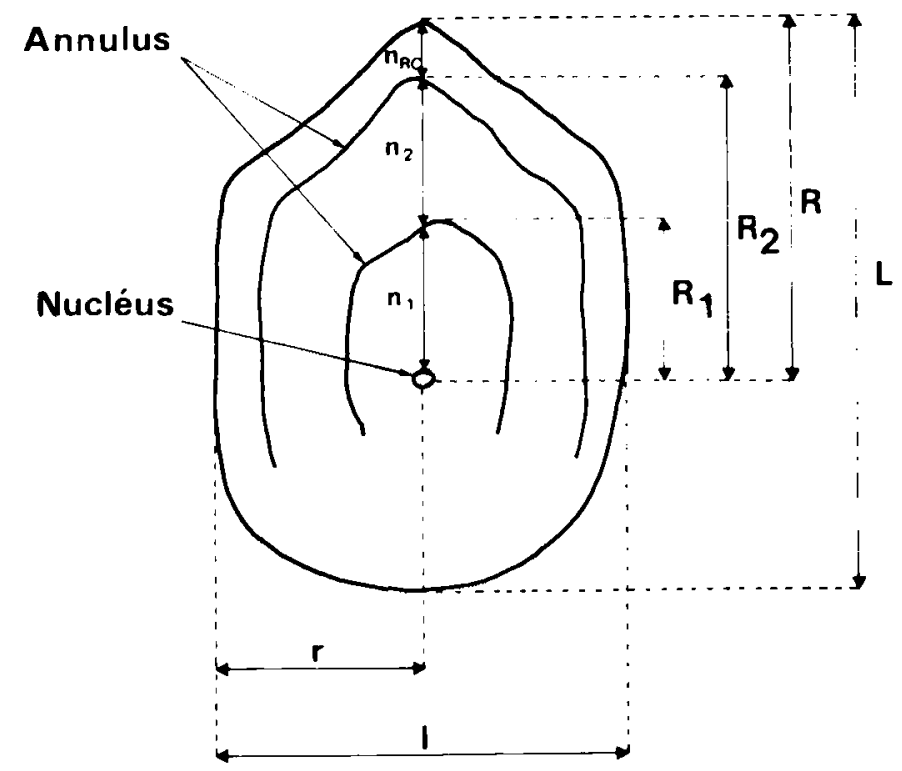

figure 3 : Différents paramètres de structure et de taille mesurés sur les écailles de juvéniles de truite de mer.

$R_{1}$ et $R_{2}$ : Rayons antérieurs de l'écaille aux $1^{\text {er }}$ et $2^{\text {nd }}$ annulus

$R \quad$ : Plus grand rayon antérieur de l'écaille

n1 : Nombre de circuli de la $1^{r e}$ année de croissance

n2 : Nombre de circuli de la $2^{e}$ année de croissance

nac : Nombre de circuli après le dernier annulus

figure 3 : Various parameters of structure and size, measured on young sea trouts'scales

$R_{1}$ and $R_{2}$ : Lengths of anterior radius at the first and second annulus

$R \quad$ : Greater anterior radius'length

n1 : Number of circuli in the first growth year

$\mathrm{n}_{2} \quad:$ Number of circuli in the second growth year

nac : Number of circuli after the last annulus 
Pour 14 poissons issus de l'échantillon ( 6 àgés de $1^{+}$an et 8 de 2 ou $2^{+}$ans) et pour seulement 8 sites $\left(B_{2}, B_{3}, C_{1}, C_{2}, C_{3}, D_{2}\right.$, $D_{3}$ et $\left.E\right)$ d'autres paramètres sont mesurés:

$R_{1}$ : rayon longitudinal jusqu'au $1^{\text {er }}$ annulus

$R_{2}$ : rayon longitudinal jusqu'au 2 nd annulus

n1 : nombre de circuli pendant la $1^{1 *}$ année de croissance

n2 : nombre de circuli pendant la 2nde année de croissance printanière).

nRC : nombre de circuli du dernier annulus au bord de l'écaille (reprise de croissance

\subsection{Critères scalimétriques caractérisant les sites de prélèvement}

En utilisant la moyenne des mesures effectuées sur les 3 écailles de chaque site, on calcule pour chaque poisson de longueur fourche Lf $(e n \mathrm{~mm})$ les caractéristiques scalaires suivantes:
I/L
$R / L$
$r / I$
L/If
I/Lf
R/Lf
$r / L f$
$\mathrm{R}_{1} / \mathrm{n}_{1}$
$\left(\mathrm{R}_{2}-\mathrm{R}_{1}\right) / \mathrm{n}_{2}$
coefficient de forme (LINDROTH, 1963; BURDAK, 1986)
indicateur du degré d'allongement de la partie antérieure de l'écaille indicateur de l'asymétrie de l'écaille par rapport au grand axe : longueur totale relative
largeur totale relative
: longueur relative du plus grand rayon longitudinal
largeur relative du plus grand rayon transversal
espace intercirculi pendant la première année de croissance
espace intercirculi pendant la seconde année de croissance
$(\mathrm{R}-\mathrm{Ri}) / \mathrm{nRc}$
espace intercirculi lors de la reprise de croissance après le dernier annulus (i).

\subsection{Traitement des données}

II est réalisé sur le miniordinateur (modèle MINI6) de chez CII Honeywell Bull du centre ENSA-INRA de Rennes. Le logiciel AMANCE est utilisé pour les analyses de variances à un facteur. Un logiciel fortran de comparaison multiple de moyennes et de classification automatique ascendante hiérarchique (BAGLINIERE E., comm. pers.) permet une meilleure confrontation des sites de prélèvement.

\section{RÉSULtATS}

\subsection{Pourcentage de régénération des écailles}

Les résultats du tableau Ia confirment le fort taux de régénération des écailles, observé chez cette espèce par BAGLINIËRE et al. (1986). Les sites situés dans la partie antérieure du corps du poisson, et notammment sur le site $E$, présentent un taux de régénération $\left(t_{3}\right)$ significativement plus faible $(\alpha=0,05)$. Les plus forts taux $(70,8 \%$ a $76,9 \%)$ s'observent sur les sites $B_{1}, B_{2}$ et $D_{3}$ (tableau Ia).

Les pourcentages $t_{1}$ de bonnes écailles (détermination de l'âge et analyses fines de structure possibles) varient de $19,6 \%$ sur le site $B_{2}$ à $46,0 \%$ sur le site $A_{3}$ (tableau Ib).

L'analyse des résultats par cohorte met en évidence que le taux de régénération t3 est significativement plus faible (environ 15\%) pour les sujets d'1 an que pour ceux âgés de 2 ans. Corrélativement, le pourcentage de bonnes écailles ( $t_{1}$ ) est plus élevé pour la plus jeune cohorte. Cependant la variabilité inter-site est globalement la même pour les deux groupes d'âge.

\subsection{Forme et taille des écailles}

\section{Forme}

Le rapport entre la plus grande largeur I de l'écaille et sa plus grande longueur $L$ a été utilisé par BURDACK (1986) pour caractériser la forme des écailles: plus le coefficient I/L est élevé plus l'écaille est arrondie. Les écailles sont significativement $(\alpha=0,05)$ plus allongées (figure 4) dans la partie postérieure du poisson (zone D), mais aussi quand elles proviennent de sites proches de la ligne latérale (zones 2 et 3 ). Le site $E$ (épaule) fait exception à cette règle car il présente des écailles aussi allongées que celles du site $\mathrm{A}_{3}$. On constate donc une variabilité longitudinale et verticale de la forme de l'écaille.

Le rapport $R / L$ est significativement équivalent $(\alpha=0,05)$ sur tous les sites pour la totalité de l'échantillon. Sa valeur moyenne de 0,538 $(\sigma=0,030)$ montre que pour les smolts de truite de mer, la partie antérieure de l'écaille est sensiblement plus grande que la partie postérieure.

De même, le rapport $r / l$ est équivalent $(\alpha=0,05)$ pour tous les sites. Sa valeur moyenne 0,524 $(\sigma=0,018)$ traduit une légère disymétrie autour du grand axe de l'écaille. 
Tableau I : Pourcentages moyens d'écailles fortement régénérées $t 3$ (a) et exemptes de toute régénération $t 1$ (b) pour 15 écailles sur différents sites du cóté gauche du corps de 34 smolts de truite de mer. Chaque site est défini par l'intersection d'une zone verticale $(A$ a $D)$ et d'une zone horizontale ( 1 a 3 ) (voir figure 1 ). Les pourcentages moyens statistiquement équivalents $(\alpha=0,05)$ sont regroupés dans un méme cadre (- - ou ........ ou -..- ou -.-.).

Table 1 : Average percentages of extremely regenerated $\mathrm{t}$ (a) and without any regeneration $t 1$ (b) of 15 scales from various areas of the body's left side for 34 smolt sea trouts. Each area is defined by crossing of vertical zone (A to D) and horizontal zone ( 1 to 3 ) (see figure 1). The average percentages which are statistically equivalent $(\alpha=0,05)$, are grouped in a same frame or or -..- or -.-.).

Ia

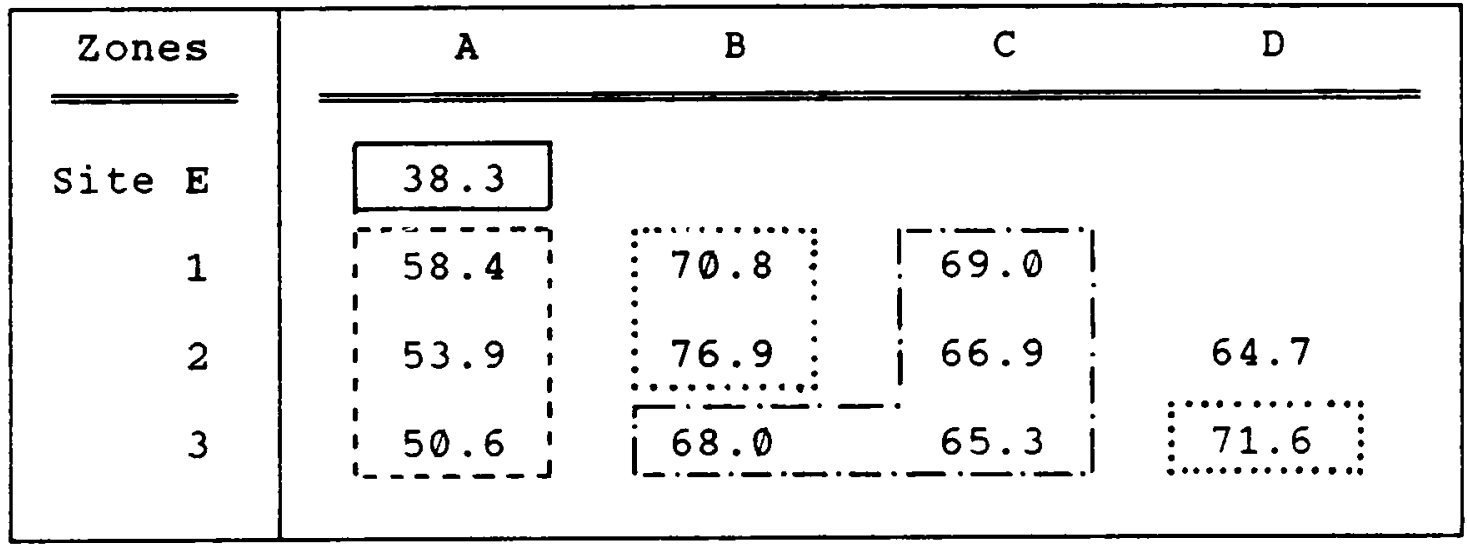

Ib

\begin{tabular}{|c|c|c|c|c|c|}
\hline \multicolumn{2}{|c|}{ Zones } & A & B & C & $D$ \\
\hline Site & E & 38.3 & & & \\
\hline & 1 & 39.2 & 25.5 & 25.7 & \\
\hline & 2 & 41.3 & 19.6 & 30.8 & 29.0 \\
\hline & 3 & 46.0 & 27.8 & 30.7 & 23.9 \\
\hline
\end{tabular}




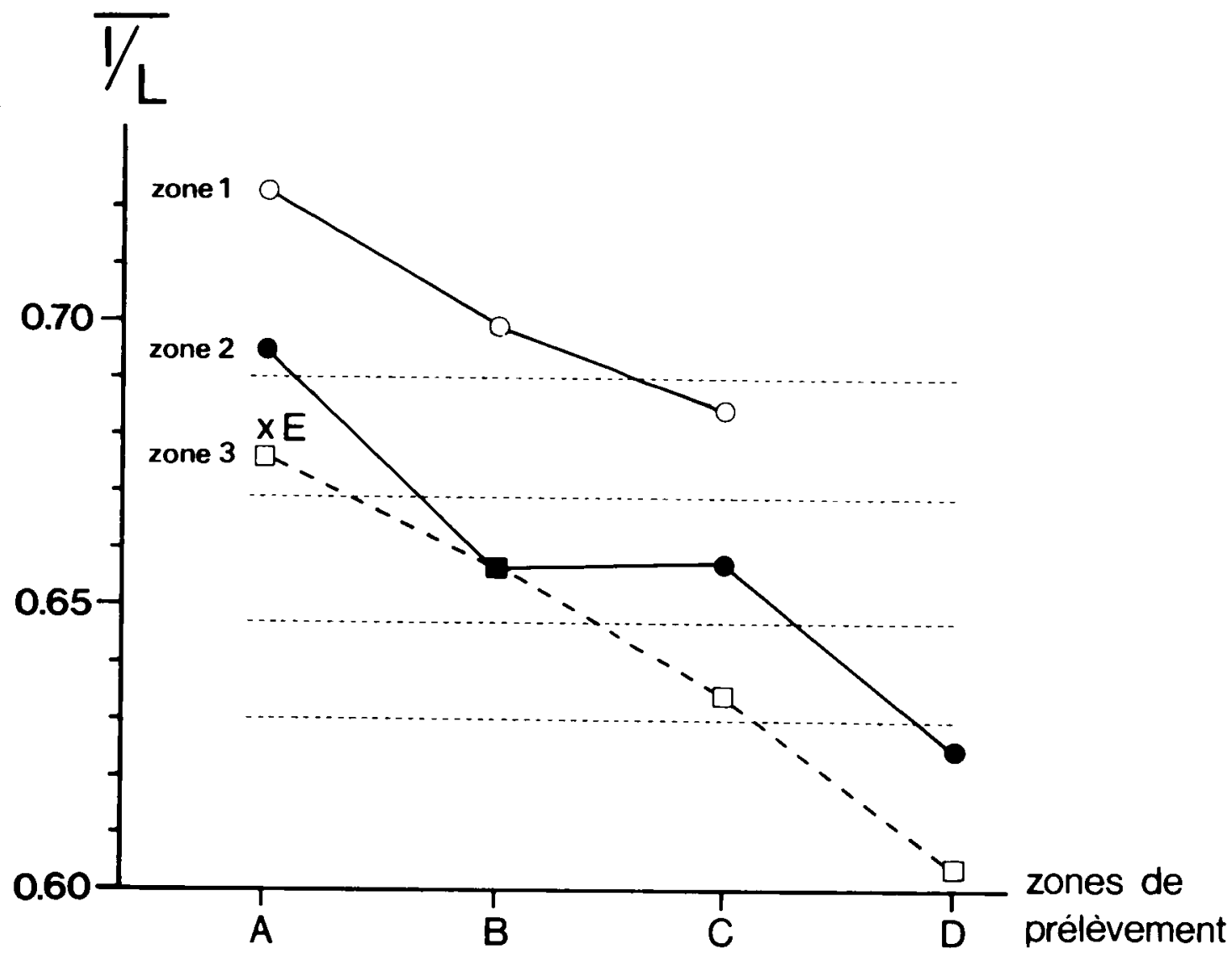

figure 4 : Variabilité du coefficient de forme moyen (I/L), des écailles de 34 smolts de truite de mer pour différents sites de prélèvement (voir figure 1)

$\mathbf{L}=$ Longueur (diamètre longitudinal) moyenne pour 3 écailles

$I=$ largeur (diamètre transversal) moyenne pour 3 écailles

figure 4 : Variability of the average form's coefficient $(I / L)$ of 34 smolt sea trouts'scales from various sampling areas (see figure 1)

$\mathbf{L}=$ mean length (longitudinal diameter) of 3 scales

I = mean width (transversal diameter) of 3 scales

Taille

Sont utilisées des mensurations relatives afin de s'affranchir de la variabilité inter-poisson de la taille des écailles : chaque mesure ( $L, R$, l et r) étant rapportée à l'unité de longueur fourche (Lf) du poisson.

La partie antérieure de l'écaille, la plus utilisée dans la détermination de l'âge et les rétromesures, a été prioritairement étudiée. L'évolution du rapport $R / L f$ selon les sites est représentée à la figure 5 (la variabilité du rapport L/Lf est semblable). Les écailles apparaissent plus longues près de la ligne latérale et en s'éloignant de la partie antérieure du poisson. Les différences induites par l'éloignement de la ligne latérale (les valeurs moyennes de R/L f de la zone 1 valent $88,8 \%$ de celles de la zone 2) sont plus importantes que celles observées sur des sites horizontaux voisins. Par exemple, les valeurs de R/Lf en zone B sont égales en moyenne à $95,8 \%$ de celles en zone $C$. Par contre, le rayon ne décroît pas entre la zone $C$ et la zone $D$.

La variabilité de la largeur des écailles, étudiée à travers les rapports $1 / L f$ et $r / L f$ (figure 6 ) est comparable avec celle observée sur les longueurs de l'écaille (R/Lf). La seule différence avec $R / L f$ réside dans le fait que la largeur diminue sensiblement entre la zone $C$ et la zone $D$.

\section{Comparaison des deux cohortes}

Pour chaque site, les diverses mensurations et coefficients de forme des écailles sont significativement équivalents pour les deux cohortes excepté le rapport $R / L$. Les valeurs de ce paramètre sont plus élevées $(\alpha=0,05)$ chez les individus de 2 ans sur les zones $B, C$ et $D$. 


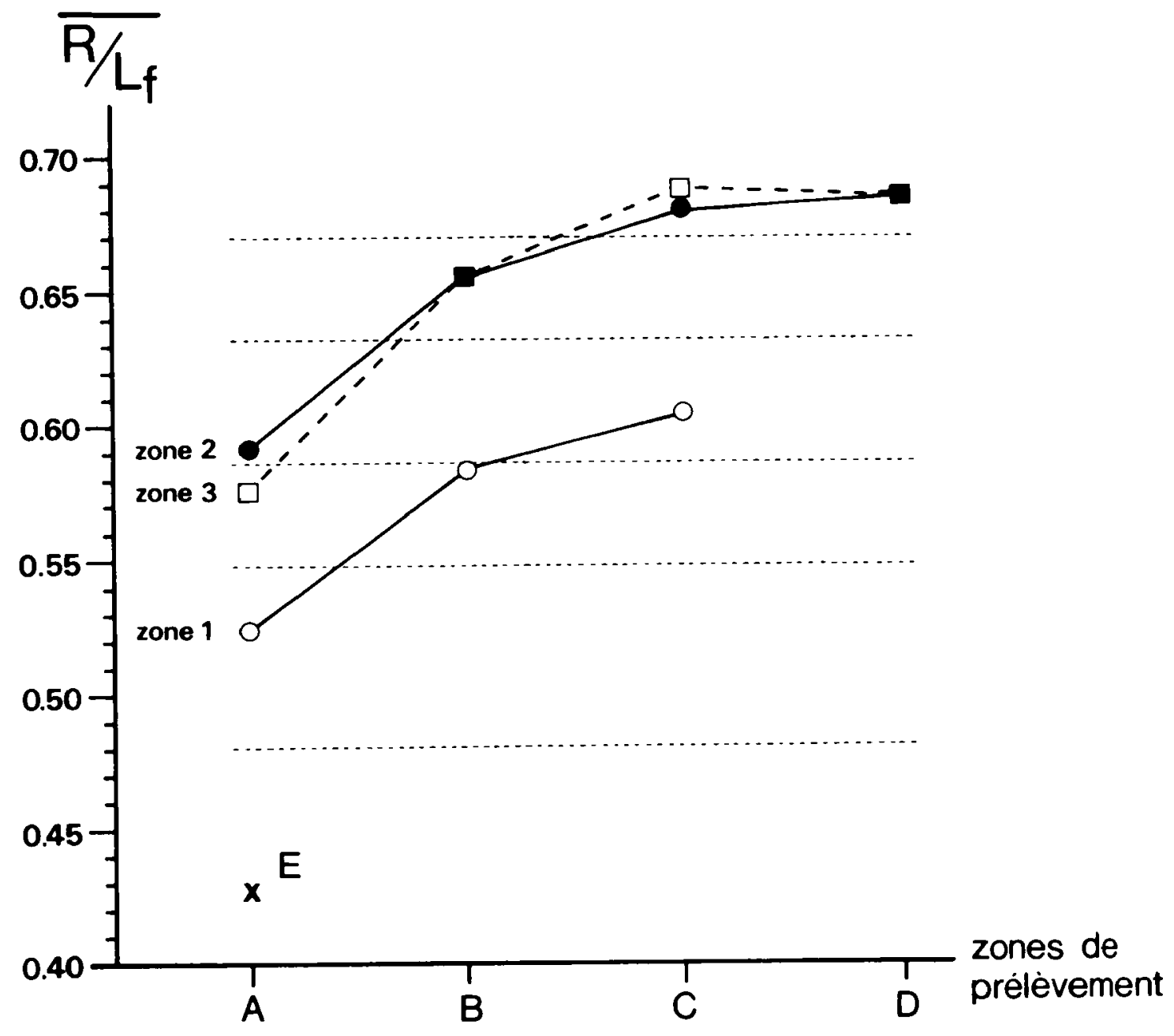

figure 5 : Variabilité du rayon antérieur relatif moyen (R/Lf) des écailles de 34 smolts de truite de mer pour différents sites de prélèvement (voir figure 1)

$\mathbf{R}=$ rayon antérieur moyen pour 3 écailles (en gradations de micromètre)

Lf = Longueur fourche du poisson en $\mathrm{mm}$

figure 5 : Variability of the average relative anterior radius (R/Lf) for 34 smolt sea trouts' scales from various sampling areas (see figure 1)

$R=$ mean anterior radius for 3 scales (in gradations of micrometer)

Lf $=$ fork length of the fish in $\mathbf{m m}$ 


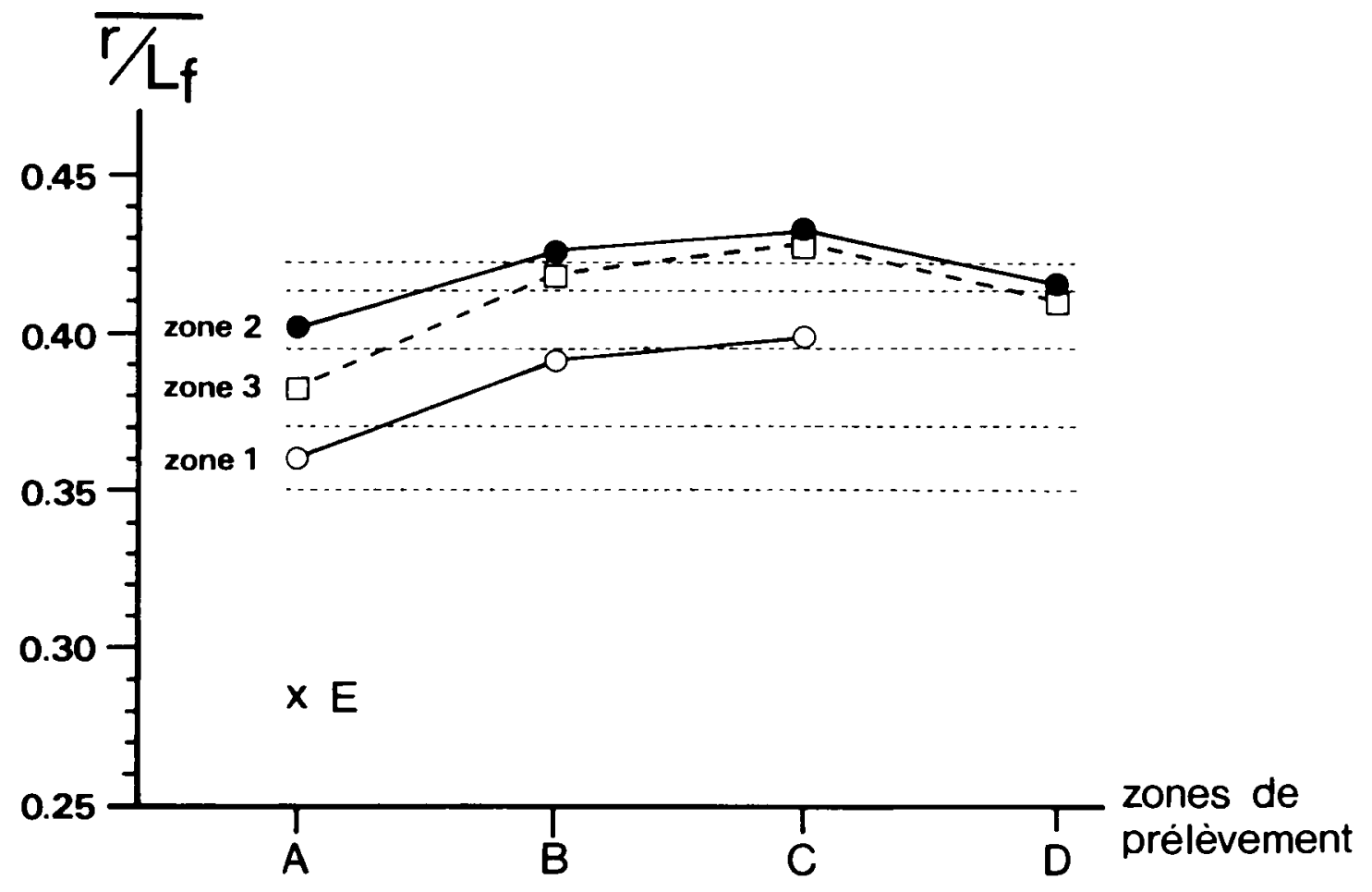

figure 6 : Variabilité du rayon transversal relatif moyen $(r / L f)$ des écailles de 34 smolts de truite de mer pour différents sites du corps (voir figure 1)

$r$ = rayon transversal moyen pour 3 écailles (en gradations de micromètre) Lf = longueur fourche du poisson en $\mathrm{mm}$

figure 6 : Variability of the average relative transversal radius $(r / L f)$ for 34 smolt sea trouts'scales from various sampling areas (see figure 1)

$r=$ mean transversal radius for 3 scales (in gradations of micrometer)

Lf $=$ fork length of the fish in $\mathrm{mm}$

\subsection{Nombre de circuli et espace intercirculi}

Pour mettre en évidence la variabilité du nombre de circuli et de l'espace intercirculi selon les sites de prélèvement, les deux cohortes de l'échantillon et les années de croissance ont été analysées séparément.

\section{Nombre de circuli}

Quelle que soit la cohorte considérée, le nombre de circuli (tableau 2) est statistiquement le même pour tous les sites excepté pour le site $E$ (Epaule) où ils sont moins nombreux. Cette différence est d'ailleurs plus accentuée pour la première année de croissance ( $\left.n_{1}\right)$. En fait, le nombre de circuli d'une écaille n'étant pas fonction de l'âge mais de la longueur du poisson (ALVORD, 1954), la variabilité inter-poisson peut masquer dans les résultats la variabilité inter-site. En effet, bien que statistiquement non significative, s'observe une diminution du nombre de circuli pour la première année de croissance entre le site $\mathrm{C}_{2}$ (rangs 1 à 3 au-dessus de la ligne latérale) et le site $\mathrm{C}_{1}$ (rangs 4 à 6), cette variabilité verticale tend à s'estomper lors de la seconde année de croissance (différence du nombre de circuli du site $\mathrm{E}$ par rapport aux autres sites).

En outre, les résultats mettent en évidence que le nombre de circuli pour la première année de croissance n'est pas nettement inférieur à celui de la seconde année pour l'échantillon de poissons âgés de 2 ans.

\section{Espace intercirculi}

La distance entre les circuli, pouvant être considérée comme un indice de lisibilité de l'écaille, est calculée globalement par année de croissance. Les différences entre les sites sont moins 
Tableau II : Nombre moyen de circuli $\left(n_{i}\right)$ et son écart type $\left(\sigma_{n i}\right)$ par année de croissance i pour deux cohortes de smolts de truite de mer en différents sites du corps (voir figure 1).

Table II : Average number of circuli $\left(n_{i}\right)$ and its standard error $\left(\sigma n_{i}\right)$ for each growth year $i$ for two smolt sea trouts'cohort from different body's areas (see figure 1).

\begin{tabular}{|c|c|c|c|c|c|c|}
\hline \multirow[t]{2}{*}{ Sites } & \multicolumn{6}{|c|}{ 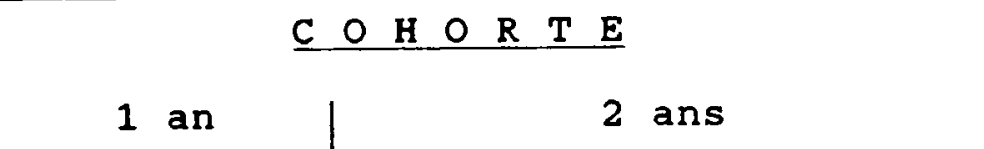 } \\
\hline & $\mathbf{n}_{1}$ & $\sigma \mathrm{n}_{1}$ & $\mathbf{n}_{1}$ & $\sigma n_{1}$ & $n_{2}$ & $\sigma \quad n 2$ \\
\hline $\begin{array}{l}\mathbf{E} \\
C_{1} \\
B_{2} \\
C_{2} \\
D_{2} \\
B_{3} \\
C_{3} \\
D_{3}\end{array}$ & $\begin{array}{l}17,0 \\
25,8 \\
27,0 \\
27,3 \\
26,5 \\
27,2 \\
26,8 \\
27,3\end{array}$ & $\begin{array}{l}3,2 \\
6,0 \\
5,9 \\
5,8 \\
6,3 \\
5,4 \\
5,8 \\
6,4\end{array}$ & $\begin{array}{l}13,4 \\
21,1 \\
22,8 \\
23,0 \\
23,6 \\
21,3 \\
22,8 \\
23,5\end{array}$ & $\begin{array}{l}3,7 \\
3,8 \\
2,9 \\
4,8 \\
4,7 \\
4,8 \\
4,5 \\
4,5\end{array}$ & $\begin{array}{l}19,3 \\
24,0 \\
23,5 \\
25,5 \\
25,5 \\
24,3 \\
27,4 \\
26,0\end{array}$ & $\begin{array}{l}3,8 \\
4,8 \\
3,8 \\
5,1 \\
4,0 \\
5,5 \\
5,3 \\
5,1\end{array}$ \\
\hline
\end{tabular}

marquées et moins systématiques que pour les autres critères (tableau 3). Néanmoins les sites $E$ et $C_{1}$ présentent généralement des espaces intercirculi statistiquement plus faibles qu'ailleurs. Pour le stade juvénile chez la truite de mer, il semble donc que la lisibilité des écailles diminue sensiblement quand on s'éloigne de la ligne latérale.

Chez les sujets âgés de 2 ans, l'espace intercirculi de la seconde année de croissance est plus grand que celui de la première année, pour tous les sites. Enfin, lors de la reprise de croissance printanière, précédant la dévalaison, l'espace intercirculi présente un net accroissement tant chez les smolts d'âge $1^{+}$, que chez ceux d'âge $2^{*}$. II est du même ordre de grandeur pour les deux cohortes.

Tableau III : Espaces intercirculi moyens $\bar{x}$ (en nombre de gradations de micromètres; 500 gradations $=1 \mathrm{~cm}$ ) et leurs écarts type $\sigma \bar{x}$ par année de croissance pour i poissons appartenant à deux cohortes de smolts de truite de mer en différents sites du corps (voir figure 1).

Table III : Mean intercirculi spaces $\bar{x}$ (in number of micrometer's gradations; 500 gradations $=$ $1 \mathrm{~cm}$ ) and standard error $\sigma \bar{x}$ for each growth year, and for $i$ fishes of the two smolt sea trout's cohorts from various body's areas (see figure 1).

\begin{tabular}{|c|c|c|c|c|c|c|c|c|c|c|}
\hline \multirow{4}{*}{ sites } & \multicolumn{10}{|c|}{$\subseteq O H O R T E S$} \\
\hline & \multirow{2}{*}{\multicolumn{2}{|c|}{$\begin{array}{l}1 \\
\qquad i=6\end{array}$}} & & & \multirow{2}{*}{\multicolumn{2}{|c|}{$\begin{array}{l}\text { lère année } \\
i=8\end{array}$}} & \multirow{2}{*}{\multicolumn{2}{|c|}{$\begin{array}{l}2 \text { ans } \\
\text { 2ème année } \\
i=8\end{array}$}} & & \\
\hline & & & \multicolumn{2}{|c|}{$\begin{array}{c}\text { Reprise } \\
\text { printaniere } \\
i=6\end{array}$} & & & & & \multicolumn{2}{|c|}{$\begin{array}{c}\text { Reprise } \\
\text { printaniere } \\
i=5\end{array}$} \\
\hline & $\overline{\bar{x}}$ & $\sigma \vec{x}$ & $\bar{x}$ & $0 \ddot{x}$ & $\bar{x}$ & $a \bar{x}$ & $\bar{x}$ & $\sigma \bar{x}$ & $\bar{x}$ & $\sigma \bar{x}$ \\
\hline $\begin{array}{l}E \\
C_{1}\end{array}$ & $\begin{array}{l}2.69 \\
2.69\end{array}$ & $\begin{array}{l}0.12 \\
0.09\end{array}$ & $\begin{array}{l}3,07 \\
4,07\end{array}$ & $\begin{array}{l}0.69 \\
0.61\end{array}$ & $\begin{array}{l}2,53 \\
2,51\end{array}$ & $\begin{array}{l}0.33 \\
0.21\end{array}$ & $\begin{array}{l}2,66 \\
2,80\end{array}$ & $\begin{array}{l}0.14 \\
0.21\end{array}$ & $\begin{array}{l}3,30 \\
3,77\end{array}$ & $\begin{array}{l}0.68 \\
0.37\end{array}$ \\
\hline $\begin{array}{l}\mathrm{B}_{2} \\
\mathrm{C}_{2} \\
\mathrm{D}_{2}\end{array}$ & $\begin{array}{l}2,88 \\
2,84 \\
2,94\end{array}$ & $\begin{array}{l}0.09 \\
0.13 \\
0.10\end{array}$ & $\begin{array}{l}4.15 \\
4.24 \\
4.17\end{array}$ & $\begin{array}{l}0.58 \\
0.76 \\
0.54\end{array}$ & $\begin{array}{l}2.53 \\
2.52 \\
2.56\end{array}$ & $\begin{array}{l}0.19 \\
0.23 \\
0.21\end{array}$ & $\begin{array}{l}3.01 \\
3.03 \\
2.89\end{array}$ & $\begin{array}{l}0.31 \\
0.31 \\
0.22\end{array}$ & $\begin{array}{l}4.23 \\
4.38 \\
3,58\end{array}$ & $\begin{array}{l}1.42 \\
0.79 \\
0.46\end{array}$ \\
\hline $\begin{array}{l}B_{3} \\
C_{3} \\
D_{3}\end{array}$ & $\begin{array}{l}2,77 \\
2,90 \\
2,85\end{array}$ & $\begin{array}{l}0.13 \\
0.13 \\
0.08\end{array}$ & $\begin{array}{l}4.02 \\
4.11 \\
3.75\end{array}$ & $\begin{array}{l}0.66 \\
0.65 \\
0.55\end{array}$ & $\begin{array}{l}2.62 \\
2.51 \\
2.48\end{array}$ & $\begin{array}{l}0.22 \\
0.17 \\
0.16\end{array}$ & $\begin{array}{l}2,94 \\
2,94 \\
2,81\end{array}$ & $\begin{array}{l}0.16 \\
0.22 \\
0.17\end{array}$ & $\begin{array}{l}4,25 \\
3,82 \\
3,59\end{array}$ & $\begin{array}{l}1.45 \\
0.35 \\
1.00\end{array}$ \\
\hline
\end{tabular}




\subsection{Tailles rétrocalculées}

La variabilité inter-site des tailles rétrocalculées par le modèle de LEA a été analysée (tableau 4).

Pour les deux cohortes, les tailles rétrocalculées à 1 an $\left(L_{1}=L f \times R_{1} / R\right)$ sont plus faibles sur le site $E$. Cette différence n'est pas statistiquement $(\alpha=0,05)$ significative, la variabilité inter-poisson masquant très probablement encore une fois la variabilitè inter-site. Les tailles rétrocalculées à 2 ans $\left(L_{2}=L f \times R_{2} / R\right)$ pour les smolts de 2 ans sont comparables sur les 8 sites de prélèvement étudiés.

Tableau IV : Tailles fourches moyennes (en $\mathrm{mm}$ ) rétrocalculées par le modèle de LEA à 1 an $\left(L_{1}\right)$ et 2 ans ( $\left.L_{2}\right)$ et les écarts types associés ( $\sigma L_{1}$ et $\sigma L_{2}$ ) pour deux cohortes de smolts de truite de mer, en différents sites du corps (voir figure 1).

Table IV : Mean fork lengths (in $\mathrm{mm}$ ) back calculated by LEA's model at 1 year ( $\left.L_{1}\right)$ and 2 years (L2) and their standard error ( $\sigma L_{1}$ and $\sigma$ L2) for two smolt sea trouts' cohorts, at various body's areas (see figure 1).

\begin{tabular}{|c|c|c|c|c|c|c|}
\hline \multirow[t]{2}{*}{ Sites } & \multicolumn{6}{|c|}{$\begin{array}{llllllll}C & O & H & O & R & T & E & S \\
\end{array}$} \\
\hline & $\mathrm{L}_{1}$ & $0<1$ & $\mathbf{L}_{1}$ & 011 & $\mathrm{~L}_{2}$ & $0 \quad 22$ \\
\hline $\begin{array}{l}E \\
C_{1} \\
B_{2} \\
C_{2} \\
D_{2} \\
B_{3} \\
C_{3} \\
D_{3}\end{array}$ & $\begin{array}{l}114 \\
122 \\
123 \\
122 \\
122 \\
122 \\
121 \\
124\end{array}$ & $\begin{array}{l}18 \\
25 \\
24 \\
25 \\
26 \\
25 \\
24 \\
28\end{array}$ & $\begin{array}{l}78 \\
88 \\
89 \\
84 \\
87 \\
86 \\
83 \\
87\end{array}$ & $\begin{array}{r}14 \\
17 \\
8 \\
17 \\
18 \\
18 \\
18 \\
17\end{array}$ & $\begin{array}{l}199 \\
198 \\
198 \\
197 \\
197 \\
196 \\
199 \\
194\end{array}$ & $\begin{array}{l}20 \\
21 \\
20 \\
19 \\
20 \\
23 \\
21 \\
22\end{array}$ \\
\hline
\end{tabular}

\section{DISCUSSION - CONCLUSION}

\section{Variabilité inter-site des structures scalaires}

La variabilité de taille (rayons, diamètres) et de forme (I/L) des écailles observées chez les smolts de Truite de mer est en accord avec les résultats obtenus sur des saumons du genre Oncorhynchus (SCARNECCHIA, 1979; BILTON, 1984) ou du genre Salmo (MARTYNOV, 1983). Les écailles les plus longues se situent sur la partie postérieure du poisson (zone $C$ et $D$ ) et près de la ligne latérale. Les écailles les plus larges sont localisées toujours près de la ligne latérale, mais seulement dans la zone $C$. Ce phénomène peut s'expliquer par les fluctuations dans le temps de la morphologie des poissons. LINDROTH (1963) évoque ainsi la "plasticité" des écailles qui s'adaptent dans leur croissance à la surface de scalation. Les écailles disposeraient de moins de largeur pour se développer à proximité du pédoncule caudal (zone D) qu'au milieu du corps du poisson (zone B et C). II résulte notamment de ces considérations que les écailles ayant la forme la plus allongée se situe dans la zone $\mathrm{D}$ près de la ligne latérale comme cela a été montré chez certains cyprinidés par BURDACK (1986).

Comme chez le Saumon atlantique (MARTYNOV, 1983), la variabilité verticale est plus importante que la variabilité horizontale. En effet, les différences de taille (rayons, diamètres) ou de forme $(1 / L)$ sont plus grandes entre les rangs 1-3 et 4-6 que entre deux zones horizontales voisines de la partie postérieure du corps (zones B, C, D). Cela peut s'expliquer par l'apparition des premières écailles près de la ligne latérale (SIRE, 1981), mais aussi par le fait que, après un arrêt temporaire de la croissance du poisson, la formation de nouveaux circuli au bord de l'écaille débute près de la ligne latérale (MARTYNOV, 1983).

Dans cette étude, la diminution du nombre de circuli de la partie antérieure du poisson vers le pédoncule caudal et de la ligne latérale vers le dos du poisson, observée par SCARNECCHIA (1979), MARTYNOV (1983) ou BILTON (1984), n'a pas été statistiquement mise en évidence. La variabilité inter-site est sans doute masquée par la variabilité inter-poisson compte tenu du mode de traitement 
statistique des données. De même, l'espace intercirculi, indice de lisibilité des écailles, est comparable pour tous les sites sauf $E$ et $C_{1}$ (les plus éloignés de la ligne latérale dans l'échantillon) où ils sont plus faibles. SCARNECCHIA (1979) n'a pas mis en évidence une telle variation entre les sites proches de $\mathrm{C}_{1}$ et $\mathrm{C}_{2}$ chez le saumon coho (Oncorhynchus kisutch).

Enfin, une symétrie est mise en évidence entre les 3 rangs de part et d'autre de la ligne latèrale pour la plupart des caractéristiques des écailles, comme cela a été signalé pour le Saumon atlantique (REDDIN, 1982 in PREVOST, 1987).

\section{Variabilité dans le temps}

La structure de l'écaille évolue avec l'âge du poisson, avec en particulier un allongement préférentiel de la partie antérieure de l'écaille qui confirme les observations faites par LINDROTH (1963) sur le "déplacement" du nucleus suite à une croissance inégale des parties antérieures et postérieures. Si l'espace intercirculi s'accroît entre la première et la seconde année de croissance. l'augmentation du nombre de circuli, observée par BEALL (1979) sur les écailles de Truites des Kerguelen, n'a pas été mise en évidence. Des changements de structure mais aussi de forme des écailles avec l'âge, sont mentionnées chez le Saumon atlantique par MARTYNOV (1983) et LINDROTH (1963) qui concluent que l'allométrie de croissance chez les poissons affecte aussi les écailles. Ces considérations ont amené CARLANDER (1985) à proposer l'utilisation d'une relation entre le rapport $\mathrm{R} / \mathrm{I}$ (et non le rayon $\mathrm{R}$ ) et la longueur du poisson dans le rétrocalcul des tailles.

Seulement pour certains critères tels le nombre de circuli et l'espace intercirculi et le rétrocalcul des tailles (modèle de LEA), la variabilité entre les sites s'avère plus importante lors de la première année de croissance pour l'échantillon de juvéniles de Truite de mer àgés de 2 ans. Chez le Saumon atlantique, LINDROTH (1963) et surtout MARTYNOV (1983) ont montré ce phénomène pour des critères de taille et de structure des écailles. Quant aux tailles rétrocalculées par le modèle de LEA, PREVOST (1987) a mis en évidence, toujours pour cette espèce, qu'elles étaient plus faibles surtout pour la première année d'eau douce lorsque l'on utilisait les écailles de l'épaule. La plus grande variabilité des rayons lors de la première année de croissance s'explique de nouveau par l'apparition non simultanées des écailles sur toute la surface du poisson (LINDROTH, 1963).

Tous ces résultats confirment le mode de scalation décrit chez la truite commune ( $S$. trutta L.) par PAGET (1920), PAROTT (1933) et SETNA (1934) (in SIRE, 1981) qui ont observé que la mise en place des écailles débutait dans la partie postérieure du corps, légèrement en arrière de la nageoire dorsale et le long de la ligne latérale. On peut supposer que, comme chez le Saumon sockeye (BILTON, 1984) le développement des écailles chez les juvéniles de Truite de mer se poursuit le long de la ligne latérale vers l'avant et l'arrière puis dorsalement et ventralement.

\section{La zone optimale de prélèvement}

Quelle que soit l'espèce considérée, la meilleure zone de prélèvement des écailles correspond à celle où ces structures sont apparues en premier. Les écailles y sont donc les plus grosses (les plus longues et les plus larges) et possèdent le plus grand nombre de circuli (BILTON, 1984). De plus, le choix doit aussi porter sur une zone à bonne lisibilité des écailles (espace intercirculi élevé) et pour laquelle il n'y a pas de sous-estimation des tailles rétrocalculées. Enfin le pourcentage d'écailles utilisables pour la détermination de l'âge $\left(t_{1}+t_{2}\right)$ et pour des études fines de structure $\left(t_{1}\right)$ doit évidemment être pris en compte.

Pour chaque paramètre caractérisant les sites de prélèvement d'écailles étudiés, chez les smolts de truite de mer, trois classes de "qualité" ont été retenues: bonne (+), moyenne (0) et mauvaise (-) (tableau V). Bien qu'aucun site ne soit "parfait", il résulte de cette analyse que les sites $C_{2}, C_{3}$ et $D_{2}$ puis les sites $B_{2}, B_{3}$ et $D_{3}$ sont inclus dans la zone susceptible d'être proposée pour le prélèvement des écailles chez la truite de mer.

Pour les critères de taille et de structure des écailles, les sites de la partie antérieure du poisson (zone A) sont à proscrire, de même que ceux localisés à plus de trois rangs au-dessus de la ligne latérale (zone 1 et site E) (figure 7). Ces résultats sont comparables à ceux obtenus sur les Saumons (genres Salmo et Oncorhynchus).

La zone où l'on trouve les meilleures écailles se situe donc:

- près de la ligne latérale, dans les trois rangs au-dessus (SCARNECCHIA, 1979; MARTYNOV, 1983) mais aussi dans les trois rangs en-dessous.

- entre l'arrière de la nageoire dorsale et l'avant de l'anale (zone C) (MARTYNOV, 1983). Cependant l'extension horizontale des limites jusqu'au milieu de la dorsale (zone B) vers l'avant et jusqu'à l'avant de l'adipeuse (zone D) vers l'arrière est envisageable. 
Tableau V : Qualité (bonne $=+;$ moyenne $=0$; mauvaise $=-$ ) des écailles de juvéniles de truite de mer pour différents critères (régénération, taille, structure, rétrocalcul des longueurs par le modèle de LEA) en différents sites du corps (voir figure 1).

t 3 (Régén.) = pourcentage d'écailles largement régénérées.

1 (Bonnes) $=$ pourcentage de bonnes écailles.

Table V : Quality (good $=+;$ mean $=0$; bad $=-$ ) of young sea trout scales for many orteria (gegeneration, size, structure, back calculation of length by LEA's model) from various body's areas (see figure 1 ).

13 (Régén.) = proportion of great regenerated scales.

1 (Bonnes) $=$ proportion of good scales.

\begin{tabular}{|c|c|c|c|c|c|c|c|}
\hline \multirow[b]{2}{*}{ Sites } & \multicolumn{2}{|c|}{ Régénération } & \multicolumn{2}{|c|}{ Taille } & \multicolumn{2}{|c|}{ Structure } & Rétro- \\
\hline & $\begin{array}{c}\text { t } 3 \\
(\text { Régen.) }\end{array}$ & $\begin{array}{c}t \quad 1 \\
\text { (Bonnes) }\end{array}$ & Longueur & Largeur & $\begin{array}{r}\text { Nombre } \\
\text { circuli }\end{array}$ & $\begin{array}{l}\text { Espace } \\
\text { Inter- } \\
\text { circuli }\end{array}$ & \\
\hline $\begin{array}{l}E \\
A_{1}\end{array}$ & $\begin{array}{l}+ \\
+\end{array}$ & + & - & - & - & - & - \\
\hline$A_{2}$ & + & + & 0 & 0 & & & \\
\hline $\mathrm{A}_{3}$ & + & + & 0 & 0 & & & \\
\hline$-B_{1}$ & - & - & 0 & 0 & & & \\
\hline $\bar{B} 2 !$ & - & - & + & + & + & + & + \\
\hline$-\mathrm{B}_{3} !$ & 0 & - & + & + & + & 0 & + \\
\hline $\overrightarrow{\mathrm{C}}_{1}$ & 0 & - & 0 & 0 & + & - & + \\
\hline $\mathrm{C}_{2}$ & 0 & 0 & + & + & + & + & + \\
\hline $\mathrm{C}_{3}$ & 0 & 0 & + & + & + & + & + \\
\hline $\mathrm{D}_{2}$ & 0 & 0 & + & + & + & + & + \\
\hline $\mathrm{D}_{3}$ & - & - & + & + & + & 0 & + \\
\hline
\end{tabular}

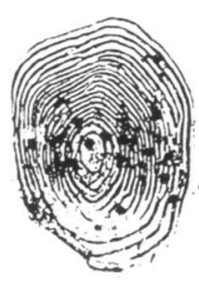

$0,5 \mathrm{~mm}$

Site $E$
Site $\mathrm{C}_{2}$

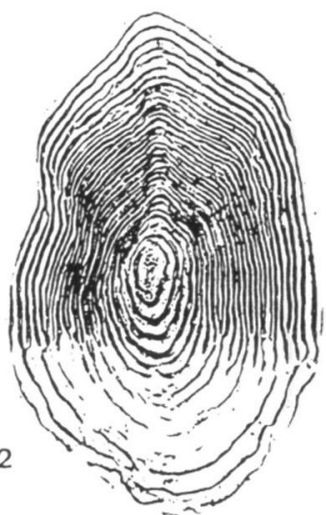

figure 7 : Ecailles d'un smolt de truite de mer âgés de 1 + an (longueur fourche $=143 \mathbf{m m}$ ), prélevées sur les deux sites du corps $\mathrm{E}$ et $\mathrm{C}_{2}$ (voir figure 1)

figure 7 : Scales of a smolt sea trout, 1 year old (fork length $=143 \mathrm{~mm}$ ), sampled ont the two body's areas $E$ and $C_{2}$ (see figure 1)

Dans un souci de rapprochement avec les normes définies pour le Saumon atlantique (ANONYME, 1984) la zone proposée pour le prélèvement des écailles chez les juvéniles de truite de mer, se situerait alors dans les trois premiers rangs au-dessus ou en-dessous de la ligne latérale, et de part et d'autre d'une droite joignant l'arrière de la nageoire dorsale et l'avant de la nageoire anale. Cela correspond à une zone centrée horizontalement sur les sites $C_{2}$ et $C_{3}$ du présent travail. Des taux de régénération "inacceptables" (ANONYME, 1984) dans les trois premiers rangs au-dessus de la ligne latérale ont conduit le groupe de travail de lecture d'écaille du Saumon atlantique du CIEM à étendre la zone standard de prélèvement chez cette espèce aux rangs 1 à 6 (BAGLINIËRE, 1986); voire à la restreindre aux rangs 3 à 6 (ANONYME, 1984). Au vu des présents résultats, une démarche similaire n'est pas envisageable pour la truite de mer: d'une part, les taux de régénération apparaissent équivalents $(\alpha=0,05)$ pour les rangs 1-3 (zone 2) et pour les rangs 4-6 (zone 1) tandis que d'autre part plusieurs critères de forme et de structure des écailles diffèrent nettement entre ces deux zones. En tout état de cause, le pourcentage élevé d'écailles régénérées aux abords de la ligne latérale, amène à proposer chez la truite de mer un prélèvement minimum de 10 à 15 écailles permettant d'en obtenir au moins trois utilisables à des fins de lecture d'âge et d'études fines. L'extension de la zone de prélèvement pourrait se faire horizontalement en cas d'absence d'écailles sur l'emplacement proposé. 


\section{Perspectives}

Afin de pouvoir déterminer et choisir au niveau international une zone standard de prélèvement des écailles pour la truite de mer, des travaux complémentaires restent nécessaires pour:

- confirmer les présents résultats à travers une étude similaire sur le stade adulte (OMBREDANE et RICHARD, données non publiées); toutefois, chez le Saumon atlantique, la variabilité inter-site des caractéristiques des écailles concerne surtout la phase juvénile en eau douce (PREVOST, 1987).

- obtenir des résultats sur des stocks à plus faible croissance. Il a en effet été montré tant chez S. salar (MARTYNOV, 1983) que chez $S$. trutta (BEALL et DAVAINE, 1988) que le nombre de circuli formés dépendait du taux de croissance annuel de l'individu (lié pour une grande part au facteur température); chez des individus à faible taux de croissance, l'absence du premier annulus a ainsi été constatée sur les écailles provenant de la partie antérieure du poisson ou de zones éloignées de la ligne latérale (MARTYNOV, 1983).

\section{BIBLIOGRAPHIE}

ALVORD W., 1954. Validity of age determinations from scales of brown trout, rainbow trout, and brook trout. Trans. Amer. Fish. Soc., 83, 91-103.

ANONYME, 1984. Atlantic Salmon scale Reading. I.C.E.S., Aberdeen 23-28 April, 15 p.

BAGLINIERE J.L., 1985. La détermination de l'âge par scalimétrie chez le Saumon atlantique (Salmo salar) dans son aire de répartition méridionale: utilisation pratique et difficultés de la méthode. Bull Fr. Pêche, Piscic., 298, 69-105.

BAGLINIERE J.L., MAISSE G., 1985. Precocious maturation and smoltification in wild atlantic Salmon in the Armorican massif, France. Aquaculture, 45, 249-263.

BAGLINIERE J.L., LECLERC G., RICHARD A., 1986. Comparaison entre l'âge et la croissance déterminés par scalimétrie et otolimétrie chez la truite de mer (Salmo truttaL.). Bull. Fr. Pêche Piscic., 301, 56-66.

BEALL E., 1979, Analyse scalimétrique d'une population de Truite de mer, Salmo truttaL., des lles Kerguelen, TAAF : structure d'âge, croissance, reproduction. Thèse Doc. Biol. Anim. (Hydrobiologie), Fac. Sci. Toulouse, $182 \mathrm{p}$.

BEALL E., DAVAINE P., 1988. Analyse scalimétrique de la truite de mer (Salmo trutta L.) : formation des anneaux et critères d'identification chez les individus sédentaires et migrateurs d'une même population acclimatée aux îles Kerguelen (TAAF). Aquat. Living Resour., 1, 3-16.

BILTON H.T., 1984. Variation in scales sampled from different body areas of Sockeye Salmon yearlings (Oncorhynchus nerka). Can. Tech. Rep. Fish. Aquat. Sci., no 1297, 11 p.

BURDAK V.D., 1986. Morphologie fonctionnelle du tégument écailleux des poissons. Cybium, 10, $n^{\circ} 3$ (Supplément), 1 vol., $147 \mathrm{p}$.

CARLANDER K.D., 1949. Some considérations in the use of fish growth data based upon scale studies. Trans Amer. Fish. Soc., 79, 187-194.

CARLANDER K.D., 1985. Sampling problems in deriving a body scale regression for growth calculation from fish scale. Verh. Internat. Verein. Limnol., 22, 2534-2536.

DAVAINE P., BEALL E., 1982. La truite de mer Salmo trutta L. 1758. Bibliographie. Bull. Sci. et Tech. du départ. d'Hydrobiologie de l'INRA, $\mathrm{n}^{\circ} 10,53 \mathrm{p}$.

FAHY E., 1983. Characteristics of Riverine phase of large Sea Trout. Salm. Trout Magaz., 225, 66-69.

FOURNEL Françoise, EUZENAT G., FAGARD J.L., 1987. Rivières à truites de mer et à saumons de Haute Normandie. Réalités et perspectives. Le cas de la Bresle. 315-325, In: THIBAULT M. et BILLARD R., Restauration des rivières à Saumon, Ed. INRA (Paris).

HESTHAGEN T., 1985. Validity of age determination from scales of brown trout (Salmo trutta L.). Inst. Freshwat. Res. Drotting-holm Rep., 62, 65-70.

HILE R., 1970. Body-scale relation and calculation of growth in fishes. Trans. Amer. Fish. Soc., 3 , 468-474.

JARVI T.H., MENZIES W.J.M., 1934. Report of the meeting of Salmon and Trout experts. Rap. proc. Verb. CIEM., 91, 3-10.

KIPLING C., 1962. The use of the scales of the trout (Salmo trutta L.) for the back calculation of growth. J. Cons. Int. Explor. Mer, XXVII, 3, 304-315.

LE CREN E.D., 1985. The biology of the sea trout, Summary of Symposium 24-26 octobre 1984. Plas Menai, Atlantic Salmon trust, $42 \mathrm{p}$. 
LINDROTH A., 1963. The body/scale relationship in Atlantic Salmon (Salmo salar L.) a preliminary report. J. Cons. int. Expl. Mer, 28, (1), 137-152.

MARTYNOV V.G., 1983. On variability of scale characteristics in Atlantic salmon (Salmo salar L.). ICES. Anadromous and catadromous Fisch. Comm., CM 1983/M:5, 21 p.

PREVOST E., 1987. Les populations de Saumon atlantique (Salmo salar L.) en France : description, relation avec les caractéristiques des rivières, essai de discrimination. Thèse de DocteurIngénieur (Halieutique) de l'Ecole Nat. Sup. Agron. de Rennes, 103 p.

REDDIN D.G., 1981. Stock indentification. ICES Anadromous and Catadromous Fish. Comm, C.M., $1981 / \mathrm{M}: 2,33-42$.

RICHARD A., 1986. Les populations de truite de mer (Salmo trutta L.) des rivières Orne et Touques (Basse Normandie) : Scalimétrie, Sexage. Caractéristiques biométriques et démographiques. Thèse $3^{\circ}$ cycle, Univ. Rennes l, 54 p.

SCARNECCHIA D.L., 1979. Variation of scale characteristics of Coho Salmo with sampling location on the body. The Progressive Fish Culturist, 41, (3), 132-135.

SIGLER W.F., 1951. Age and growth of the brown trout, Salmo trutta fario Linnaeus, in Logan river, Utah. Trans. Amer. Fish. Soc., 81, 171-178.

SIRE J.Y., 1981. La scalation (Apparition et mise en place des écailles) chez Hemichromis bimaculatus (GILL, 1862) (Teleosteens, Perciformes, Cichlidés), Cybium, $3^{\mathrm{e}}$ Série, 5, 51-66.

SYCH R., 1967. Dependence of scale radius and body weight on length of sea trout (Salmo trutta L.) from vistula river. Roczn. Nauk. Roln., H 90, (2), 327-339.

SYCHR., 1983. Attemps of using the scale characteristics for separation of some Baltic salmon and sea trout stocks, ICES, Baltic Salmon working Group., CM 1983/M : 9, 43.83. 